\title{
DÉFICITS NEUROPSICOLÓGICOS ASOCIADOS A ALTERACIONES CEREBRALES SECUNDARIAS A TRATAMIENTOS ONCOLÓGICOS
}

\author{
COGNITIVE DEFICITS ASSOCIATED TO BRAIN CHANGES SECONDARY TO \\ ONCOLOGICAL THERAPIES
}

\author{
Magda Gómez-Cruz \\ Calidad de Vida. Centro de Rehabilitación Neuropsicológica
}

Resumen

Gracias a recientes avances en cirugía, radiación y farmacología se está incrementando la tasa de supervivencia al cáncer pero cada vez es más frecuente encontrar personas que presentan alteraciones cognitivas y comportamentales posteriores a tratamientos de quimioterapia y radioterapia. Estas alteraciones pueden relacionarse con lesiones causadas al tejido cerebral, especialmente a la sustancia blanca, por tales tratamientos oncológicos. En este trabajo se exponen los procesos neurobiológicos que subyacen a los mencionados déficits neuropsicológicos, como son la inhibición de la neurogénesis del hipocampo, la destrucción de los precursores de oligodendrocitos y los cambios producidos en la sustancia blanca. Se examina de qué manera todo ello se expresa en la forma de fallas en atención, velocidad de procesamiento de la información, memoria, y funciones ejecutivas.

Palabras clave: Cáncer. Déficits cognitivos. Leucoencefalopatía. Neurogénesis. Quimioterapia. Radioterapia. Sustancia blanca.

\section{Abstract}

Recent advances in surgery, radiation and pharmacology have brought an increase on the rate of survival from cancer. However, it is frequent to see patients affected by cognitive impairments and behavioral changes after chemotherapy and radiology treatments. These changes might be related to injury to the brain tissue, especially to the white matter, caused by such oncological therapies. This work examines the neurobiological processes that underlie the mentioned neuropsychological impairments, such as the hippocampal neurogenesis inhibition, the destruction of oligodendrocytes precursors, as well as changes in white matter. Cognitive dysfunction sequelae in the form of deficits on attention, speed of information processing, memory, and executive functions are also studied.

Key words: Cancer, Cognitive impairment, chemotherapy, leukoencephalopathy, Neurogenesis, radiotherapy, white matter.

\section{Correspondencia:}

Magda Gómez-Cruz

Calidad de Vida, Centro de Rehabilitación Neuropsicológica

Calle 106 No. 54-93 cons. 701 Bogotá,

Colombia

E.mail: neuropsicologia@une.net.co 


\section{INTRODUCCIÓN}

Estudios recientes indican que el uso sistemático e intensivo de drogas quimioterapéuticas, la irradiación, o la combinación tan generalizada de las dos para intentar la cura de diversos tipos de cáncer, no solamente del sistema nervioso central (SNC), da lugar a efectos neurológicos adversos a largo y a corto plazo ${ }^{(1,2)}$. La toxicidad que estos agentes químicos causan a las células cerebrales y los eventos desencadenados por la radiación son comunes, sin importar el tipo de cáncer que se esté tratando, trayendo consigo daño a las células progenitoras responsables de la neurogénesis hipocámpica y de la integridad de la sustancia blanca (SB). Estas alteraciones se reflejan en diversos déficits neuropsicológicos, especialmente en atención, velocidad de procesamiento, memoria y funciones ejecutivas, afectando la calidad de vida de los supervivientes ${ }^{(3-5)}$. Se han estudiado los efectos de la quimioterapia y la radioterapia sobre el tejido cerebral y el funcionamiento cognitivo posteriores a la resección de tumores cerebrales por esos métodos ${ }^{[6-9)}$, en pacientes con cáncer del seno ${ }^{(10-14)}$, de testículos y de ovario ${ }^{(15,16)}$, y otros de cáncer de pulmón ${ }^{(17)}$, en los que los pacientes han desarrollado cambios en sus funciones mentales seguidos al tratamiento de quimio y radioterapia, o a la combinación de los dos. También se han visto casos infantiles de leucemia o de neoplasias cerebrales tratados con radiación en que se afectan las funciones cognitivas y el desarrollo (por disfunción endocrina) ${ }^{(18,19)}$.

Parece ser que los fármacos utilizados en quimioterapia hacen un mayor daño a las células normales que a las cancerosas. Se ha comprobado muerte de los precursores neuronal, glial (especialmente de los oligodendrocitos), supresión o reducción de la división de las células progenitoras (la que es vital para los procesos repara- dores y la repoblación neuronal), y disfunción en la diferenciación celular ${ }^{(1,2)}$. La irradiación, por su parte, provoca procesos necróticos y leucoaraiosis ${ }^{(59,60)}$. Estos efectos post-tratamiento además de implicar alteraciones de la neurogénesis hipocámpica y lesiones en la sustancia blanca ${ }^{(20-22)}$, pueden redundar en leucoencefalopatía, epilepsia, o infarto cerebral ${ }^{(1,2,23)}$. Todas estas son secuelas que pueden extenderse a largo plazo y hacerse evidentes inclusive años después del tratamiento contra el cáncer en la forma de déficits cognitivos y resultan por demás devastadores tanto para el desarrollo en niños y adolescentes, como para los procesos reparadores en adultos ${ }^{(1,24)}$.

En el presente trabajo se examinan los procesos biológicos desencadenados por los efectos neurotóxicos de los agentes quimioterapéuticos más comunes y los necróticos provocados por la irradiación. Se estudian las dos hipótesis neuropsicológicas más conocidas sobre los déficits cognitivos asociados a tratamientos anticáncer como son la de la alteración de la neurogénesis del hipocampo, y la de la destrucción de los precursores de oligodendrocitos y los consiguientes cambios producidos en la sustancia blanca. Se ve también de qué manera todo ello se expresa en la forma de deterioro cognitivo progresivo.

\section{NEUROTOXICIDAD DE LOS AGENTES QUIMIOTERAPÉUTICOS Y EFECTOS DE LA IRRADIACIÓN}

Los fármacos más conocidos que en altas dosis y casi siempre en combinación con radioterapia craneal pueden producir daño a cualquier parte del sistema nervioso central son el BCNU o carmustine, cisplatin, cytarabine, 5-fluorouacil, ifosfamide, L-aspariginase, methotrexate, procarbazine, corticosteroides, y algunos modificadores de las respuestas biológicas como el 
interferón y el interleukin-2. La aplicación de manera intratecal o intracarotidea produce mayores efectos neurológicos que la terapia oral o intravenosa ${ }^{(1,2,25)}$. Estos agentes citotóxicos en altas dosis vulneran las células neurales progenitoras e interfieren en la proliferación y diferenciación de las células gliales, especialmente de los oligodendrocitos que son los encargados de la formación de mielina ${ }^{(26)}$.

La radioterapia, por su parte, puede causar complicaciones en una etapa temprana o en una etapa tardía y éstas consisten primordialmente en radionecrosis o leucoaraiosis, leucoencefalopatía, y por lo tanto, disfunción cognitiva. La potencialidad del daño se incrementa con la combinación con quimioterapia en altas dosis $y$ en especial con ciertos medicamentos usados como el cisplatin y el methotrexa$\mathrm{te}^{(27-29)}$.

\section{Vulnerabilidad de las células neurales progenitoras y de los recursores de oligodendrocitos a los fármacos usados en quimioterapia}

A pesar de que no existe una gran cantidad de investigaciones sobre la neurotoxicidad de la quimioterapia como sí lo hay y desde hace mucho sobre las consecuencias de la radioterapia, es bien sabido los efectos nefastos de la aplicación de methotrexate directamente en los ventrículos cerebrales que produce dilatación, edema y se destruye la capa celular ependimaria que los rodea. Si se aplica cytarabine a la superficie cerebral se causan efectos negativos a las células subventriculares en sus procesos de división ${ }^{(30)}$. Los oligodendrocitos constituyen otro de los principales blancos de destrucción de los agentes farmacológicos empleados en la quimioterapia y son especialmente vulnerables al carmustine (BCNU), rutinariamente usado para combatir tumores cerebrales y los linfomas de Hodgkin y no Hodgkin ${ }^{(31)}$.
La inhibición de la generación de precursores de oligodendrocitos a partir de progenitoras neurales o la limitación de la diferenciación hacia oligodendrocitos maduros resulta especialmente grave ya que se impide el proceso de producción de la mielina, capa lipídica que envuelve la mayoría de los axones del SNC y que favorece la propagación de los potenciales acción. Los oligodendrocitos durante la diferenciación hacen que se aumente la actividad de la gliserol-3fosfato deshidrogenasa y de la fosfodiesterasa, enzimas relacionadas con la mielogénesis, y una vez diferenciados expresan la proteina proteolipídica y la MPB o proteina básica de la mielina ${ }^{(32)}$. $\mathrm{Si}$ sus precursores son atacados o si no hay diferenciación hacia oligodendrocitos no hay mielinización y la transmisión del impulso nervioso se ve disminuida o anulada, sobreviniendo la "desconexión" de los fascículos axonales que se refleja en deterioro cognitivo, como lo veremos más adelante en el apartado relacionado con el daño a la sustancia blanca.

Dietrich et al. ${ }^{(1,2)}$ en su estudio pionero en este campo, encontraron que las células neurales progenitoras y los precursores de oligodendrocitos son altamente vulnerables a los agentes más comúnmente usados en la quimioterapia como el BCNU (carmustine), que se usa rutinariamente en el tratamiento de tumores cerebrales, mieloma y linfomas, al cisplatin y al cytarabine. Este es el primer estudio que lo demuestra tanto in vitro como in vivo. El nitrosurea del BCNU causa entrecruzamiento entre la guanina y la citosina, como ha sido comprobado por dicho grupo, al aplicar BCNU y cisplatin sobre células madre neuroepiteliales humanas, (las que generan todo tipo de células del SNC), y células neurales progenitoras y precursoras de rata. Según estas investigaciones, cuando los agentes quimioterapeúticos mencionados se aplicaban en ratas se observaba un aumento de la muerte celular y un decremento en 
la división celular en la zona subventricular, el giro dentado, el hipocampo y el cuerpo calloso, efecto que se prolongaba aún semanas después de la administración del fármaco. Estos autores también analizaron células humanas cancerosas de útero, seno, colon, ovario, meningioma y glioma para establecer in vitro cómo las células progenitoras son más vulnerables a dichos agentes quimioterapeúticos que las mismas células cancerosas. Pequeñas concentraciones de cisplatin, $1 \mu \mathrm{M}$, o de BCNU, $25 \mu \mathrm{M}$, reducían la viabilidad de las células precursoras de oligodendrocitos $y$ astrocitos (O-2A/OPCs) entre un 60 y un $90 \%$, mientras que las células tumorales eran más resistentes y no llegaban a exceder estos niveles de vulnerabilidad a los agentes tóxicos que se les aplicaban.

De otro lado, el cisplatin es un producto alcalino que influye sobre el DNA y conduce a muerte celular por mecanismos que todavía se están investigando ${ }^{(33)}$. Por su parte, cytarabine, un antimetabolito usado en el tratamiento de la leucemia y los linfomas también mostró su toxicidad en las células progenitoras, comprometiendo la división celular en los estudios in vitro y causando muerte celular y reducción de la división in vivo. Este producto afecta también a los oligodendrocitos, los cuales no se dividen, lo que señala que esas sustancias nocivas al SNC no solamente prefieren a las células con propiedades proliferativas $s^{(1,2,34)}$.

\section{Efectos de la irradiación}

La irradiación craneal suele ser el tratamiento recomendado en los casos de tumores primarios y metastáticos del cerebro, los cánceres del cuello y de la cabeza, así como de leucemias y linfomas del SNC. La radioterapia es considerada muy recomendable también después de resección de tumores. La dosis tolerable que puede ser soportada por el tejido ce- rebral es muy limitada por lo cual es difícil evitar causar daño. Este procedimiento genera una serie de respuestas cerebrales muy complejas que incluyen el aumento de agentes oxidativos y de citocinas inflamatorias. Se produce una inhibición de la neurogénesis endógena y se altera la diferenciación de las células precursoras neurales $^{(35)}$, lo cual se asocia con alteraciones de la neurogénesis del hipocampo, uno de los mecanismos que subyacen al deterioro cognitivo progresivo que se presenta en estos sobrevivientes de cáncer ${ }^{(21,22)}$.

Estudios que se han hecho para tratar de aclarar el diagnóstico entre recidiva tumoral y efectos post-tratamiento con radiación han encontrado que como contrapartida a un mayor índice de supervivencia de los pacientes con tumores cerebrales existe con gran frecuencia lesión cerebral post-radioterapia consistente especialmente en necrosis perilesional tardía progresiva en al menos el $50 \%$ de los casos después de radiocirugía y del $65 \%$ en casos de radioterapia intersticial. Las técnicas de PET-FDG han probado ser las más apropiadas y exactas para este tipo de diagnóstico diferencial porque permite apreciar la ausencia de metabolismo y falta de captación de la glucosa, compatibles con radionecrosis $\mathrm{s}^{(7,8,36)}$. Se pueden observar dos tipos de efectos postradioterapia, uno es el daño en el tejido glial que aunque se trate con corticoterapia no mejora el pronóstico para el paciente, y el otro, son los efectos tardíos que presentan el reto para la clínica diaria de diferenciar entre la recurrencia tumoral y la radionecrosis lo que sí tendría diferente pronóstico.

A nivel radiológico y patológico la lesión al cerebro por radiación puede ocurrir en dos formas, focal y difusa. La focal incluye una gran cantidad de anormalidades desde cambios subclínicos, a veces asintomáticos detectables solamente a través de la RM, hasta evidente necrosis cerebral. La necrosis focal se presenta como una lesión 
masiva con evidencia clínica de anormalidad neurológica y presión intracraneal aumentada. A nivel microscópico, se hallan cambios vasculares y daño en la sustancia blanca que van desde desmielinización hasta necrosis coagulativa. La lesión difusa se caracteriza por el decrecimiento periventricular en atenuación de TC y señal incrementada en la densidad-protón y las imágenes de RM en T2. Los síntomas aparecen en forma de déficits en las funciones mentales que varían en latencia ${ }^{(36)}$.

En cuanto a la interferencia en la neurogénesis, se sabe que el hipocampo es uno de los dos sitios más activos para este proceso en el cerebro de los mamíferos, y la proliferación de células precursoras en la zona subgranular genera células que migran a la capa granulada y se diferencian en fenotipos neuronales y gliales de los que se originan nuevas células. Cualquier cambio en esta neurogénesis está íntimamente relacionado con cambios cognitivos, entonces los efectos de la radiación, la inflamación, y el estrés oxidativo inhiben la neurogénesis y resultan en deterioro cognitivo ${ }^{(37-40)}$. Tanto en niños como en adultos se produce una merma en el desempeño cognitivo que ha sido relacionado con esa disfunción hipocámpica que resulta del decremento en la neurogénesis inducido por la radiación. La alteración del microambiente que regula el destino de las células progenitoras y de la capacidad proliferativa de las células madre neurales, el hecho de que los precursores neurales restantes adopten formas gliales, y los cambios en la angiogénesis microvascular propia de la neurogénesis del adulto se reflejan en los mencionados déficits ${ }^{(41)}$. La aparición de los síntomas de lesión secundaria a tratamiento con radiación pude ir de algunos meses hasta años, GalarzaInza ${ }^{(42)}$, describe esos efectos adversos tardíos y los relaciona con un gran daño a la sustancia blanca con vacuolación, fibrosis, necrosis, hemorragias, calcificaciones y te- langlectasias que se pueden ligar con los efectos de la radioterapia.

Pasando a lo que se ha visto en el laboratorio, se afirma que las células progenitoras son tan sensibles a la radiación que aún una dosis baja aplicada al cráneo de una rata madura es suficiente para hacer daño a la neurogénesis del hipocampo ${ }^{(43)}$. Madsen et al. ${ }^{(44)}$, en su modelo animal, han reportado que la irradiación bloqueó la formación de nuevas neuronas en el giro dentado del hipocampo, lo que fue relacionado con el desempeño de ratas en dos ocasiones diferentes, a los ocho y a los veintiún días después de la irradiación, en dos tareas de memoria a corto plazo que diferían con respecto a su dependencia del hipocampo. Los animales en los que se había bloqueado la neurogénesis presentaron resultados más pobres que los controles en una tarea de reconocimiento que implicaba el hipocampo, lo que apoya la idea de que la generación de nuevas neuronas es necesaria para que las funciones relacionadas con esa área del cerebro funcionen apropiadamente. Este modelo de irradiación craneal que imita al tratamiento para diferentes tipos de cáncer Ileva a Madsen y su grupo investigador a sugerir que el bloqueo de la neurogénesis contribuye a los efectos cognitivos del tratamiento, tales como déficits de memoria, disforia y letargia.

En otro trabajo, Panagiotakos et al. ${ }^{(45)}$, administraron radiación a cerebros de ratones en una dosis de $25 \mathrm{G}$, protegiendo el bulbo olfatorio para efectos compensadores de la pérdida neuronal, y llevaron a cabo análisis de los daños sufridos en diferentes regiones, durante quince meses. De manera inmediata se produjo la supresión de la neurogénesis y de la proliferación en la zona subventricular. Se presentó una pérdida del antígeno oligodendroglial O4 y los progenitores NG2 se vieron limitados en su proliferación, y a los nueve meses de la radiación ya se veía una desmielini- 
zación difusa en todos los cerebros. A los doce meses las pruebas de RM mostraban pérdida del volumen del cuerpo calloso y señales claras de progresiva desmielinización, la que a los quince meses ya se había extendido, además había áreas de necrosis focal. Se muestran así los efectos a largo plazo de la radiación y su conexión con el daño irreversible a las células madre en la zona subventricular (las que ya no pueden compensar la pérdida neuronal), y a los precursores de oligodendrocitos. Hay desmielinización tardía que precede a necrosis, efectos que se han observado también en muestras de SB humana tomadas postradiación.

Por su lado, Munjal et al. ${ }^{(34)}$, condujeron experimentos con ratas irradiadas para ver la posibilidad de mejoría de los déficits cognitivos tras el agotamiento de las existencias de células progenitoras en sus cerebros inducido por la radiación, especialmente en la región del hipocampo. En efecto, cuatro meses después de transplantarles células madre de embriones humanos pudieron comprobar migración hacia la zona subgranular del giro dentado y notaron una superioridad del desempeño de estos animales en tareas cognitivas dependientes del hipocampo en comparación a los sujetos que no recibieron transplante, las cuales presentaban un incremento de sus déficits de más del $50 \%$.

\section{Daño a la sustancia blanca}

La exposición de los afectados de cáncer a tratamientos químicos y radiológicos parece traer también efectos nocivos a la SB y conducir a placas de desmielinización, lesión y aislamiento de los axones, llevando a necrosis, leucoencefalopatia y leucoaraiosis ${ }^{(1,2,42)}$. La SB, vía de comunicación cerebral por excelencia, ocupa gran parte del interior del cerebro, constituye el $50 \%$ de su volumen, y está compuesta por células de la glía, vasos sanguíneos, y axones y dendritas que mediante circuitos cortos y varios fascículos mielinizados comunican distintas zonas cerebrales. Está formada por fibras nerviosas, o fascículos, que conectan sectores de un mismo hemisferio a las que se les llama de asociación, por las que comunican un hemisferio con otro o comisurales (cuerpo calloso, fornix, comisura anterior, septum), y por las de proyección que unen la corteza con núcleos grises inferiores ${ }^{(46)}$. Por lo tanto, cualquier interrupción en las conexiones de la SB causada por daño axonal, aislamiento o desmielinización traerá consecuencias a nivel cognitivo.

Esta problemática de las secuelas neurológicas post-tratamiento oncológico ha contribuido al creciente interés actual por el estudio de la sustancia blanca y de las patologías que trae su lesión. Después de décadas de concentrarse en el estudio de la sustancia gris, los neurocientíficos le han dado ahora la importancia que tiene la $\mathrm{SB}$, pues muchos de los déficits en las funciones cognitivas que se presentan tras una lesión o patología cerebral pueden relacionarse con fenómenos histopatológicos como el deterioro y desconexión de los conductos de la SB, la desmielinización de los cilindros axonales, así como de la degradación de las células gliales, astrocitos y especialmente oligodendrocitos ${ }^{(47-51)}$.

Se ha comprobado a través de resonancia magnética y espectroscopia que los cambios en la SB inducidos por tratamientos antineoplásicos se producen hasta en un $70 \%$ de las personas, aún varios meses después de su aplicación y en diversos grados de leve a severo ${ }^{(1,52)}$. Por medio de las mismas neuroimágenes se han encontrado cambios anatómicos en sobrevivientes de cáncer tales como volúmenes más pequeños de sustancia blanca y gris en diferentes regiones del cerebro un año después de tratamiento con quimioterapia, comparado con grupo control, lo que obviamente se correlacionaba con el bajo rendimien- 
to en pruebas cognitivas ${ }^{(11)}$. También se ha reportado pérdida del volumen cerebral y atrofia hipocampal asociados a tales tratamientos ${ }^{(21,53)}$. Sin embargo, como las lesiones difusas de los axones no son fácilmente detectadas por las técnicas de neuroimágen corrientes se han diseñado otras como el DTI (diffussion tensor imaging) para medir la integridad de la SB, el cual evalúa la integridad de los axones in vivo y ayuda a establecer si los déficits cognitivos tienen que ver con su lesión ${ }^{(54)}$. En general, hasta ahora con una técnica o con otra, los hallazgos post-tratamiento oncológico de daño en la SB se diagnostican como leucoencefalopatía y como leucoaraiosis, veamos entonces en qué consisten estas patologías cuando son secundarias a las terapias anticáncer.

\section{Leucoencefalopatía latrogénica}

La leucoencefalopatía hace referencia a atrofia cerebral y es un hallazgo común posradiación ${ }^{(36)}$. En su forma necrotizante es el daño difuso a la sustancia blanca que sigue a la quimioterapia, ya sea aplicada sola o en combinación con radioterapia. En los dos casos se producen secuelas de tipo cognitivo. León González ${ }^{(6)}$, se refiere a "leucoencefalopatía yatrógena" cuando se produce evolución satisfactoria pero se presentan alteraciones severas tardías de las funciones psíquicas superiores y del comportamiento poniendo en evidencia la neurotoxicidad en el sistema nervioso central causado por el tratamiento oncoespecífico. Es decir, que el mismo tratamiento que erradica el trastorno inicial provoca alteraciones que conducen a leucoencefalopatía y consiguiente deterioro cognitivo.

Duffner $^{(19)}$ recoge los resultados de varios estudios realizados desde los años 1970s sobre la influencia de la quimioterapia sola y en combinación con radiación en la aparición de leucoencefalopatía en niños afectados por leucemia, sin tumores del SNC, para ver los efectos adversos de los tratamientos sobre su declive intelectual. En un principio se compararon niños que habían sido irradiados con niños a los que se les había inyectado Methotrexate, notándose un descenso en el coheficiente intelectual (IQ) de los primeros, entonces esto Ilevó a la tesis de que la quimioterapia sola era segura, que no producía efectos nocivos en el área intelectual, creencia que prevaleció por casi tres décadas. Esta idea fue reforzada por estudios con sujetos que habían recibido la combinación de las dos terapias y posteriormente fueron diagnosticados con leucoencefalopatía y mostraban en la TAC calcificaciones en los ganglios basales, atrofia cerebral e hipodensidad en áreas de SB, en oposición a otro grupo de niños que solamente habían recibido quimioterapia (Methotrexate) y no radiación. Pero las cosas cambiaron cuando en 1997 se vio el deterioro cognitivo inducido por la quimioterapia en un grupo de niños con leucemia sin relación con el SNC y que no habían recibido terapia de radiación. La evidencia de leucoencefalopatía se obtuvo con neuroimágenes de RM y fue concomitante con los cambios cognitivos observados en ese grupo.

Estos hallazgos fueron corroborados por posteriores estudios, entre ellos uno que se llevó a cabo en Alemania con 23 niños aquejados de meludoblastoma que fueron tratados con altas dosis de Methotrexate y con inyección intraventricular de este mismo fármaco, pero sin radiación, de los cuales solamente cuatro no desarroIlaron leucoencefalopatía, además se vio la correlación entre el grado de ésta y la dosis total aplicada intraventricularmente. Estos chicos mostraron una media de IQ mucho más baja que el grupo control pero mejor que otro grupo que había pasado por irradiación. Se ha mostrado entonces a través de estudios como éstos que la quimioterapia o la irradiación solas o en combinación son capaces de provocar 
daño a la SB y por consiguiente disfunción cognitiva.

\section{Leucoaraiosis inducida}

Algunos autores utilizan el término leucoaraiosis inducida al hablar de los hallazgos hechos en los cerebros de los pacientes sometidos a sesiones de quimioterapia y radioterapia ${ }^{(55)}$. Leucoaraiosis es un término radiológico con el que se denomina la rarefacción de la SB, lesiones periventriculares o subcorticales que se aprecian como hipodensas en la TC e hiperintensas en la RM en T2, y que se asocian a deterioro cognitivo. La isquemia crónica parece ser el origen de este fenómeno de leucoaraiosis, en la forma de alteraciones en la microcirculación cerebral, lo que lleva a la desmielinización ${ }^{(56)}$. Se afectan las células de la glia y no las neuronas porque sus axones son más vulnerables y se localizan próximos a zonas de vasos sanguíneos, por ello en caso de escaso flujo sanguíneo son los primeros en verse afectados por hipoxia(57).

El funcionamiento vascular normal implica que desde las principales arterias cerebrales a través de pequeñas arteriolas que forman un complejo sistema microvascular la sangre llegue a la SB, pero el estrechamiento de esas arteriolas, provocado por la toxicidad de los tratamientos oncológicos, hace que de manera crónica se disminuya el flujo sanguíneo. Esta hipoperfusión y la hipoxia concomitante serían los responsables de la aparición de leucoaraiosis. La leucoaraiosis se considera un proceso gradual sostenido en el tiempo. La hipótesis de cómo la mencionada hipoperfusión va dañando la SB plantea que la isquemia leve pero prolongada favorece la defosforilización de la proteina TAU, la cual es muy importante para los microtúbulos que es por donde se mueve la cinecina /dineina para transportar el ácido ribonucléico que codifica las proteinas necesarias para la producción de mielina, y los ribosomas que al ubicarse cerca de los terminales de los oligodendrocitos Ilevan a cabo la síntesis de mielina. Al interrumpirse este proceso por hipoperfusión e hipoxia hay un déficit en esa síntesis de mielina, hay demielinización, y al hacerse más grave la hipoxia hay muerte de células gliales y mayor demielinización de la $\mathrm{SB}^{(58,59)}$.

En la leucoaraiosis periventricular se afectan principalmente las fibras largas de asociación que conectan la corteza con zonas subcorticales pero no incide sobre las fibras en " $U$ " de conexión intercortical ${ }^{(60)}$. Las alteraciones cognitivas que acompañan a la leucoaraiosis varían según el volumen de SB afectada y su localización, y pueden ir desde un mínimo déficit cognitivo hasta la demencia ${ }^{(61)}$. Se ha notado enlentecimiento en la velocidad de procesamiento, fallas en fluidez verbal y memoria (lesión del área periventricular), y alteración en las funciones ejecutivas la que estaría relacionada con una desconexión cortical con áreas subcorticales o con otras regiones lejanas de la corteza. Los problemas a estos dos niveles tendrían que ver con fallas en tareas que involucren memoria y capacidades visuoespaciales y visuoconstructivas ${ }^{(57)}$.

\section{DÉFICITS COGNITIVOS QUE SOBREVIENEN A LAS LESIONES CEREBRALES RELACIONADAS CON TRATAMIENTOS ONCOLÓGICOS}

La agresividad de los tratamientos oncológicos que puede traer como resultado la desaparición del cáncer se deja sentir también en los síntomas adversos que los pacientes experimentan en sus funciones superiores. Ya durante la misma aplicación del tratamiento esas personas sienten que sus funciones cognitivas son menos eficientes, lo que antes se atribuía a tensión emocional o depresión. Pero investigacio- 
nes recientes muestran que sí desde ese mismo momento hay un efecto perjudicial del tratamiento sobre el cerebro ${ }^{(13,62)}$. Estos síntomas iniciales y los que aparecen tardíamente en forma de fallas de atención y memoria, disminución de la capacidad de aprendizaje, dificultades de tipo ejecutivo, o anímico como depresión, abulia o apatía, perturban la vida de los afectados y les impiden retomar normalmente sus actividades laborales, educativas o sociales. Hay entonces un espacio para la neuropsicología en toda esta problemática oncológica.

\section{Hipótesis neuropsicológicas sobre los déficits cognitivos asociados a tratamientos anti-cáncer}

Por un lado, algunos estudios se han concentrado en las dificultades de memoria y aprendizaje que se asocian con lesión al hipocampo ${ }^{(1)}$, especialmente cuando la radiación involucra los lóbulos temporales. Se sabe que el hipocampo juega un papel central en el aprendizaje a corto plazo y la memoria, que suelen ser las funciones más afectadas por la radiación, pero ahora se ha conocido también que esas funciones están influenciadas por la actividad de las células madre neurales y su capacidad proliferativa pues la capa granular del hipocampo mantiene renovándose y reestructurándose por las nuevas neuronas que se integran a ella. Según esta hipótesis, la disfunción hipocámpica acumulada que resulta de la falta de actividad normal de las células progenitoras a largo plazo, provocadas, como ya expusimos, por los agentes químicos y por la radiación, es lo que causa las dificultades de aprendizaje y memoria después de un tratamiento anticáncer $^{(43)}$. Otros autores, por su parte, sostienen que los déficits neuropsicológicos están más relacionados con el daño a la SB porque encuentran, en su opinión, que los pacientes de cáncer tienen más problemas con la recuperación de la in- formación y las funciones ejecutivas que con la consolidación, lo que hace suponer que las conexiones fronto-subcorticales de sustancia blanca serían las afectadas y no el hipocampo ${ }^{(63)}$.

De estas dos hipótesis surge lo que se puede llamar un perfil cognitivo del paciente con secuelas neuropsicológicas pasados los tratamientos en cuestión, el que se relaciona, principalmente, con fallos en la atención, enlentecimiento psicomotor, alteración de memoria y visuoespaciales, lo que explicaría los bajos niveles en tareas que impliquen estas funciones ${ }^{(57,64,65)}$. Por su parte, los cambios en la SB y las alteraciones relacionadas con los lóbulos frontales traen problemas tales como lentitud en el procesamiento de la información, fallas en fluidez verbal, funciones ejecutivas (pérdida de la capacidad de clasificación, planeación y secuenciación), todo ello manifestaciones de lo que se describe como una "desconexión" relacionada con el daño a la $\mathrm{SB}^{(59,64)}$.

En diversas investigaciones ha quedado expuesta claramente la relación entre los tratamientos oncológicos de quimioterapia y radioterapia y la aparición posterior de déficits neuropsicológicos (ver tabla 1). En el área infantil, la supervivencia en niños con tumores cerebrales se ha incrementado en los últimos 20 años pero, desafortunadamente, esas terapias empleadas sobre el sistema nervioso central en niños, particularmente la radiación, se asocian al desarrollo en ellos de problemas de aprendizaje, leucoencefalopatía, endocrinopatías y oncogénesis ${ }^{(65)}$. La leucoencefalopatía en niños con leucemia linfoblástica se manifiesta en letargia, ataques de corte epiléptico, problemas del lenguaje, ataxia y pérdida de memoria ${ }^{(66)}$.

Bernabeu et al. (2003), han revisado estudios sobre la neurotoxicidad de los tratamientos oncológicos en los que han encontrado que ésta causa cambios estructurales y muestra sus efectos tardíos en 
Tabla 1. Cambios en las funciones mentales que se pueden relacionar con tratamientos oncológicos, según estudios realizados en niños y adultos

\begin{tabular}{|l|l|}
\hline \multicolumn{1}{|c|}{ Niños } & \multicolumn{1}{c|}{ Adultos } \\
\hline- Descenso en IQ & - Enlentecimiento psicomotor \\
\hline- Problemas de aprendizaje & $-\begin{array}{l}\text { Enlentecimiento de la velocidad de procesa- } \\
\text { miento }\end{array}$ \\
\hline- Déficits en atención y memoria & - Déficits en atención \\
\hline & - Bajo rendimiento en fluidez verbal \\
\hline & - Disminución de las capacidades de aprendi- \\
& zaje \\
\hline & $-\begin{array}{l}\text { Dificultades de tipo ejecutivo (clasificación, } \\
\text { planificación, secuenciación) }\end{array}$ \\
\hline & - Bajas capacidades visoespaciales y visocons- \\
& tructivas \\
\hline
\end{tabular}

forma de alteración de las capacidades intelectuales. Ha sido reportado también por estos grupos investigadores un aumento en la supervivencia de niños con tumores malignos y con leucemia, pero de nuevo surge el problema de los efectos adversos de tales terapias sobre el SNC y del deterioro de la calidad de vida de los infantes ${ }^{(64)}$, secuelas que antes no se conocían dada la alta mortalidad por estas patologías.

En el tratamiento de las leucemias y neoplasias cerebrales infantiles se utiliza la radiación ionizante contra las células tumorales tratando de hacer el menor daño posible a los demás tejidos y la quimioterapia en la que se administran fármacos citotóxicos que como ya sabemos traen muerte celular, o una combinación de ambas terapias. La neurotoxicidad de estos agentes se revela tardíamente en estos niños y su implicación y gravedad dependerá de la edad, el tipo de neoplasia o patología, su estado pre-mórbido, y las dosis empleadas y si se aplica una sola de esas terapias oncológicas o las dos. Las alteraciones cognitivas secundarias cursan con déficit en atención, memoria y baja del cociente intelectual $(\mathrm{Cl})$. Se observa disminución del volumen de sustancia blanca que tiene que ver con los déficits atencionales ${ }^{(64,65)}$.

De otro lado, se han estudiado personas tratadas de cáncer de colon y de seno con 5-fluorouracil (5-FU) el que se asocia con daño retardado a la mielina ${ }^{(66)}$. En uno de estos estudios se encontró que el $61 \%$ de mujeres tratadas con este agente presentó un declive en atención, aprendizaje y velocidad de procesamiento que no estaban relacionados a ninguna otra causa ni variable demográfica, y que no mostraron recuperación aún un año después. La tomografía por emisión de positrones ha revelado alteraciones del metabolismo y del flujo cerebral en los ganglios basales, el giro frontal inferior y el cerebelo durante tareas de memoria en mujeres que hacía entre cinco y diez años habían sido sometidas a quimioterapia para la erradicación del cáncer de seno ${ }^{(14)}$. Igualmente se han reportado otros casos de no mejoría hasta diez años más tarde de haber terminado el tratamiento $^{(67)}$.

Se encuentran también en la literatura sobre el tema casos menos frecuentes pero igual de preocupantes, como el de hemorragias múltiples en el cuerpo calloso en pacientes de cáncer de testículos. Así 
lo revelaron las imágenes de RM, después de quimioterapia con una combinación de cisplatino (el cual ha sido asociado a complicaciones cerebrovasculares), ifosfamida y etoposido, de la que se sospecha produce una gran toxicidad en el SNC. Se presentaron depresión, desorientación y seria afectación de las funciones mentales y psicomotrices ${ }^{(15)}$.

Con base en todos estos estudios, es importante recalcar en la necesidad de la detección pronta del deterioro cognitivo en estas personas y en la implementación de un tratamiento, específicamente de estimulación de las funciones afectadas y que ayude a mantener lo conservado. No hablaríamos de rehabilitación ya que se trata de un proceso demencial ligado a los cambios neurobiológicos que ya se expusieron.

\section{CONCLUSIÓN}

Como la supervivencia al Cáncer depende de la aplicación de terapias tan agresivas, como las realizadas con fármacos altamente neurotóxicos y con radiación, desafortunadamente para el paciente hay un precio que pagar. No existen por ahora muchas alternativas para estas personas ni respuestas fáciles, se trata de lograr balancear la necesidad de sobrevivir con la calidad de vida. Mientras no se descubran terapias eficaces dirigidas únicamente al objetivo sin afectar el tejido normal, no se encuentren y pongan a disposición más agentes neuroprotectores y se ajusten los tratamientos, el uso de quimio y radioterapia, y su combinación, seguirán trayendo efectos adversos en la forma de lesión cerebral y déficits de tipo neuropsicológico para estas personas.

Ahora que se reconoce que tanto la quimioterapia como la radioterapia tienen este tipo de efectos sobre el cerebro, el desempeño cognitivo y la calidad de vida de los pacientes, resulta indispensable in- cluir a la neuropsicología dentro de las disciplinas que tienen que ver con la atención a pacientes de cáncer con el objeto de detectar tempranamente las secuelas neuropsicológicas de esos agentes químicos y radiológicos y darles un seguimiento en este sentido a las personas que reciben este tipo de tratamientos.

Por otro lado, hay todo un camino que recorrer en la línea de investigación en donde se hagan estudios longitudinales con evaluaciones neuropsicológicas previas a los tratamientos oncológicos, que sirvan de línea de base y se puedan comparar con las realizadas post-tratamiento y así llegar a conclusiones más precisas sobre los efectos adversos de los tratamientos oncológicos sobre el cerebro y las funciones mentales ${ }^{(68,69)}$. Así mismo, se podrá avanzar en el área de la Neuropsicología en la evaluación de los déficits que presentan los sobrevivientes de cáncer y en la planeación de programas de estimulación de las funciones afectadas y el mantenimiento de las conservadas.

\section{REFERENCIAS BIBLIOGRÁFICAS}

1. Dietrich J, Han R, Yang M P, Noble $M$. CNS progenitor cells and oligodendrocytes are targets of chemotherapeutic agents in vitro and in vivo. J Biol 2006; 5: 22. Doi: 101186/Jbiol50.

2. Dietrich J, kesary S. Effect of cancer treatment on neural stem and progenitor cells. Cancer Treat Res; 2009: 81-5. Doi:10.1007/ b109924_6

3. Keime-Guibert F, Napolitano M, Delattre JY. Neurological complications of radiotherapy and chemotherapy. J Neurol 1998; 245: 695-708. Doi:10.1007/s004150050271

4. Wefel J S, Kayl A E, Meyers C A. Neuropsychological dysfunction associated with cancer and cancer therapies: A conceptual review of an emerging target. $\mathrm{Br}$ J Cancer 2004; 90: 1691-6. Doi:10.1007/ s004150050271 
5. Dietrich J, Monje L, Wefel J, Meyers C. Clinical patterns and biological correlates of cognitive dysfunction associated with cancer therapy. Oncologist. 2008; 13,12: 1285-95. Doi:10.1634/theoncologist.2008-0130

6. León González R A, Areces Delgado F, Ramirez D C, Chon Rivas I, Trujillo C, Vilaú Prieto L C. Linfoma cerebral primario. Efectividad y efectos secundarios del tratamiento oncoespecífico. Rev Cubana Med. [online], 2006 Jun [citado 2011 Sep 20]; 45(2): Disponible en: http://scielo.sld.cu/ scielo.php?script=sci_arttext\&pid=S003475232006000200010\&lng=es.

7. Asensio C, Pérez-Castejón M J, Maldonado A, Montz R, Ruiz J A, Santosa M, GarcíaBerrocal A, Albert J, Carreras J L. Papel de la PET -FDG ante la duda diagnóstica de recidiva frente a radionecrosis en tumores cerebrales. Rev Neurol 1998; 27: 447-52.

8. Taylor J S, Langston J W, Reddick W E, Kingsley P B, Ogg J, Pui M H, et al. Clinical value of proton magnetic resonance spectroscopy for differentiating recurrent or residual brain tumor from delayed cerebral necrosis. Int J Radiat Oncol Biol Phys 1996; 36: 1251-61. Doi:10.1634/ theoncologist.2008-0130

9. Landier W, Kinahan K E, Shaw S, Bhatia $S$. Screening for late effects in brain tumor survivors. Cancer Treat Res 2009: 389-409. Doi:10.1007/b109924_26

10. Meyers CA, Abbruszzese J L. Cognitive functioning in cancer patients: Effect of previous treatment. Neurology 1992; 42: 434-6.

11. Inagaki M, Yoshikawa E, Matsuoka Y, Sugawara $Y$, Nakano T, Akechi T, et al. Smaller regional volumes of brain gray and white matter demonstrated in breast cancer survivors exposed to adjuvant chemotherapy. Cancer 2007; 109:146-56. Doi:10.1002/ cncr.22368

12. Stemmer SM, Stears J C, Burton B S, Jones $\mathrm{R} \mathrm{B}$, Simon J H. White matter changes in patients with breast cancer treated with high-dose chemotherapy and autologous marrow support. A M J Neuroradiol 1994; 15: 1267-73. Doi:10.1097/00002371199408000-00062

13. Wefel JS, Lenzi R, Theriault R, Davis RN, Meyers CA: The cognitive sequelae of standard dose adjuvant chemotherapy in women with breast cancer: results of a prospective, randomized, longitudinal trial. Cancer 2004, 100:2292-2299. Doi:10.1002/ cncr.20272

14. Silverman D H, Castellon S A, Lai J, Pio B S, Abraham L, Waddel P L, Phelps M R, Ganz PA. Altered frontocortical, cerebellar and basal ganglia activity in adjuvanttreated breast cancer survivors 5-10 years after chemotherapy. Breast Cancer Res Treat 2007; 103: 303-11. Doi:10.1007/ s10549-006-9380-z

15. Dietrich J, Marienhagen J, Schalke B, Bogdahn U, Schlachetzki F. Vascular neurotoxicity following chemotherapy with Cisplatin, Ifosfamide, and Etoposide. Ann Pharmacother 2004; 38 (2): 242-6. Doi: 10.1345/aph.1D106

16. Chaudhary U B, Haldas J R. Long-term complications of chemotherapy for germ cell tumours. Drugs 2003; 63 (15): 1565-7. Doi:10.2165/00003495-200363150-00004

17. Higa G M, Wise T C, Crowel E B. Severe disabling neurologic toxicity following Cisplatin treatment. Ann Pharmacoder 1995; 29 (2): 134-7.

18. Bernabeu-Verdú J, López-Luengo B, Fournier-del Castillo C, Cañete-Nieto A, SuárezRodríguez J, Castel-Sánchez B. Aplicación del Attention Process Training dentro de un proyecto de intervención en procesos atencionales en niños con cáncer. Rev Neurol 2004; 38: 482-6.

19. Duffner P K. Long-term effects of radiation therapy on cognitive and endocrine function in children with leukemia and brain tumors. Neurologist 2004; 10: 293 - 310.

20. Brown M S, Stemmer S M, Simon J H, Stears J C, Jones R B, Cagnoni P J, et al. White matter disease induced by high-dose 
chemoterapy: longitudinal study with MR imaging and proton spectroscopy. AM J Neuroradiol 1998; 19: 217-21.

21. Schneiderman B. Hippocampal volumes smaller in chemotherapy patients. Lancet Oncol 2004; 5: 202-22. Doi:10.1016/ S1470-2045(04)01443-3

22. Monje M. Cranial radiation therapy and damage to hippocampal neurogenesis. Dev Disabil Res Rev 2008; 14,3: 238-42. Doi:10.1002/ddrr.26

23. Filley C M. Toxic Leukoencephalopathy. Clin Neuropharmacol 1999; 22: 249-60.

24. Monje M, Wen P Y. Neurological complications in adults. Cancer Treat Res 2009: 113 - 132. Doi: 10.1007/b109924_8

25. Macdonald D R. Neurologic complications of chemotherapy. Neurol Clin 1991; 9: 955-67.

26. Smith J, Ladi E, Mayer-Proschel M, Noble M. Redox state is a central modulator of the balance between self-renewal and differentiation in a dividing glial precursor cell. Proc Natl Acad Sci USA 2000; 97 (18): 1003210037. Doi:10.1073/pnas.170209797

27. Guilbert F, Napolitano M, Dealattre J Y. Neurological complications of radiotherapy and chemoterapy. J Neurol 1998; 245: 695-708.

28. Shapiro W R, Young D F. Neurological complications of antineoplastic therapy. Acta Neurol Scand Suppl 1984; 100: 125-32.

29. Shih H A, Loeffler J S, Tarbell N J. Late effects of CNS radiation therapy. Cancer Treat Res 2009: 23-41.

30. Nutt C L, Noble M, Chambers A F, Cairncross J G. Differential expression of drug resistance genes and chemosensitivity in glial cell lineages correlate with differential response of oligodendrogliomas and astrocytomas to chemotherapy. Cancer Res 2000; 60: 4812-8.

31. Moore-Maxwell C A, Datto M B, Hulette C M. Chemotherapy-induced toxic leukoencephalopathy causes a wide range of symptoms: a series of four autopsies. Modern Pathology 2004; 17: 241-7. Doi:10.1038/ modpathol.3800049
32. Kumar V, Abbas A K, Fausto N, Mitchell R. Patología Humana. Madrid: Elsevier, 2008.

33. Kartalou M, Essigmann J M. Recognition of cisplatin adducts by cellular proteins. Mutat Res 2001; 478:1-21. Doi:10.1016/ S0027-5107(01)00142-7

34. Baker W J, Royer G L, Weiss R B. Cytarabine and neurologic toxicity. J Clin Oncol 1991; 9: 679-93.

35. Munjal M, Acharya A, Christieb L A, Lana M L, Donovanc P J, Cotmanb C W. et al. Rescue of radiation-induced cognitive impairment through cranial transplantation of human embryonic stem cells. Proc Natl Acad Sci U S A., 2009 November 10; 106(45): 1915019155. Doi: 10.1073/pnas.0909293106

36. Valk P E, Dillon W P, Radiation Injury of the brain. Am J Neuroradiol 1991; 12, 1: 45 - 62.

37. Mizumatsu S. Extreme sensitivity of adult neurogenesis to low doses of X-irradiation. Cancer Res 2003: 63:4021- 7 .

38. Fike J R, Rola R, Limoli C L. Radiation response of neural precursor cells. Neurosurg Clin N Am 2007; 18:115-27. Doi:10.1016/j. nec.2006.10.010

39. Rola R, Sarkissian V, Obenaus A, Nelson G A, Otsuka S, Limoli C L, Fike J R. High-LET radiation induces inflammation and persistent changes in markers of hippocampal neurogenesis. Radiat Res 2005; 164: 556560. Doi:10.1667/RR3412.1

40. Leuner B, Gould E, Shors T J. Is there a link between adult neurogenesis and learning? Hippocampus 2006; 16: 216224. Doi:10.1002/hipo.20153

41. Monje M, Mizumatsu S, Fike J R, Palmer T $D$. Irradiation induces neural precursor-cell dysfunction. NAT Med 2002, 8: 955-962. Doi:10.1038/nm749

42. Galarza-Inza J, Gómez-Suárez H, Coro-Antich R M, Domínguez-Alvarez C. Astrocitoma del cerebelo y efectos adversos de la radioterapia. Presentacion de un caso. Rev Neurol 2001; 32: 948-51.

43. Monje M L, Palmer T. Radiation injury and neurogenesis. Curr Opinion Neurol 2003; 16(2):129-34. Doi:10.1097/00019052200304000-00002 
44. Madsen $T$ M, Kristjansen $P$ E, Bolwig $T$ G, Wortwein G. Arrested neuronal proliferation and impaired hippocampal function following fractionated brain irradiation in the adult rat. Neuroscience 2003; 119 (3): 635 - 42. Doi:10.1016/S03064522(03)00199-4

45. Panagiotakos G, Alshamy G, Chan B, Abrams R, Greenberg E, Saxena A, Bradbury $M$, Edgar $M$, et al. Long-term impact of radiation on the stem cell and oligodendrocyte precursors in the brain. PLoS one 2007; 11: 2 , 7: e 588.

46. DeMyer W. Neuroanatomy. The national medical series of independent study. Media, Pa: Harwal; 1988.

47. De Carli Charles. White matter matters. Scientific American 2008; 298: 54-61.

48. Matute C, Alberdi E, Domercq M, SánchezGómez MV, Pérez-Samartín A, RodríguezAntigüedad A, et al. Excitotoxic damage to white matter. J Anatomy 2007; 210 (6): 693 -702 .

49. Guimaraes J S, Freire $M A M$, Lima R R, Souza-Rodrigues R D, Costa A M R, dos Santos C. D, et al. Mecanismos de degeneración secundaria en el sistema nervioso central durante los trastornos neuronales agudos y el daño en la sustancia blanca. Rev Neurol 2009; 48: 304-10.

50. Coleman M P, Perry V H. Axon pathology in neurological disease: A neglected therapeutic target. Trends Neurosci 2002; 25: 532-7. Doi:10.1016/S01662236(02)02255-5

51. Medana I M, Esiri M M. Axonal damage: A key predictor of outcome in human CNS diseases. Brain 2003; 126: 515-30. doi:10.1093/brain/awg061

52. Stemmer S M, Stears J C, Burton B S, Jones $\mathrm{R}$ B, Simon J H. White matter autologous marrow support. AM J Neuroradiol 1994, 15: 1267-73.

53. Madhyastha S, Somayaji S N, Rao M S, Nalini K, Bairy L. Hippocampal brain amines in methotrexate-induced Learning and memory deficit. Can J Physiol Pharmacol,
2002, 80:(11) 1076-1084. Doi: 10.1139/ y02-135.

54. Kraus M, Susmaras T, Cughlin B, Walker C, Seeney J, Little D. White matter integrity and cognition in chronic traumatic brain injury: A diffusion tensor imaging study. Brain 2007; 130, 10: 2508-19. Doi: 10.1093/brain/awm216.

55. De Luca C R, Conroy R, McCarthey M, Anderson V A, Ashley D M. Neuropsychological impact of treatment of brain tumors. Cancer Treat Res 2009: 277-96.

56. Kelley $R$ E. Ischemic demyelination. Neurol Res 2006; 28: 334 -40. Doi:10.1179/016164106X98242

57. Jiménez I, Agulla J, Pouso M., Sabucedo M., Rodríguez-Yáñez M, Sobrino T, et al. Deterioro cognitivo asociado a la leucoaraiosis: fisiopatología, manifestaciones clínicas y tratamiento Rev Neurol 2008; 47: $536-44$.

58. Szolnoki Z. Chemical events behind leukoaraiosis: medicinal chemistry offers new insight into a specific microcirculation disturbance in the brain (a chemical approach to a frequent cerebral phenotype). Curr Med Chem 2007; 14: 1027-36. Doi:10.2174/092986707780362907

59. Franco Ruíz C A. Cambios en la sustancia blanca cerebral y deterioro cognitivo. Neuropsicología Neuropsiquiatría Neurociencias 2004; 6 (1): 13 - 20.

60. Alvarez J A, Scully R E, Miller T L, Armstrong $\mathrm{F} D$, Constine L S, Friedman D, et al. Longterm effects of treatments for childhood cancers. Curr Opin Pediatr 2007; 19: 2331. Doi:10.1097/MOP.0b013e328013c89e

61. Meyers C A. How chemotherapy damages the central nervous system. J Biol 2008; 7:11, 10: 1186. Doi:10.1186/jbiol73

62. Duffner $P$ K, Cohen M E. The long-term effects of central nervous system therapy on children with brain tumors. Neurol Clin 1991; 9 (2): 479-95.

63. Gururangan S. Late effects of chemotherapy. Cancer Treat Res 2009: 43 - 65. Doi:10.1007/b109924_4 
64. Bernabeu J, Fournier C, Cañete A, LópezLuengo B, Barahona T, Grau C et al. Evaluación y rehabilitación neuropsicológica en oncología pediátrica. Psicooncología 2003; 0, 1: 117-34.

65. Bernabeu J, Fournier C, Cañete A, LópezLuengo B, Barahona T, Barreto P et al. Evaluación neuropsicológica en adolescentes oncológicos. Proyecto Unitat de oncologia pediátrica, Hospital La Fe, Valencia, España. Presentación para el $2^{\circ}$. Congreso hispano-portugués de Psicología, 2004. [online] www.uv.es/jorber2/evaluacion.

66. Han R, Yang Y M, Dietrich J, Luebke A, Mayer -Proschel $M$, Noble $M$. Systemic 5-fluoracil treatment causes a syndrome of delayed myelin destruction in the central nervous system. J Biol 2008; 7(4): 12. Doi:10.1186/jbiol69

67. Wefel J S, Lenzi R, Theriault R, Davis R $N$, Meyers $C A$. The cognitive sequelae of standard dose adjuvant chemotherapy in women with breast cancer: Results of a prospective, randomized, longitudinal trial. Cancer 2004; 100: 2292-9. Doi:10.1002/ cncr.20272

68. Matsuda T, Takayama T, Tashiro M, Nakamura Y, Ohashi Y, Shimozuma K. Mild cognitive impairment after adjuvant chemotherapy in breast cancer patients - evaluation of appropriate research design and methodology to measure symptoms. Breast Cancer 2005; 12 (4): 4, 279-87. Doi: 10.2325/jbcs.12.279.

69. Wefel JS, Vardy J, Ahles T, Schagen S B. International cognition and cancer task force recommendations to harmonise studies of cognitive function in patients with cancer Lancet Oncol; 12(7):703 -8. Doi: 10.1016/ S1470-2045 (10) 70294-1 
